\title{
VICISITUDES DE LA IMPLANTACIÓN DE LA MEDIACIÓN EN ESPAÑA
}

\section{VICISSITUDES OF THE IMPLEMENTATION OF MEDIATION IN SPAIN}

Francisco Jesús CANO VERA ${ }^{1}$

Ilustre Colegio de Abogados de Jaén, España

Recibido: 24 Abril 2021 / Aceptado: 29 Abril 2021

\section{Resumen}

En el presente artículo trataré de dar una pincelada sobre la presencia de la mediación en nuestra sociedad y, sobre todo, su implantación en nuestro sistema, judicial y extrajudicial, de resolución de conflictos, con ocasión de la reciente publicación del Anteproyecto de Ley de Medidas de Eficiencia Procesal del Servicio Público de Justicia.

Palabras clave: Mediación, Implantación social, Regulación, Requisito de procedibilidad

\begin{abstract}
In this article I will try to give a brushstroke on the presence of mediation in our society and, above all, its implementation in our system, judicial and extrajudicial, of conflict resolution, on the occasion of the recent publication of the Draft Law on Measures of Procedural Efficiency of the Public Justice Service.
\end{abstract}

Keywords: Mediation, Social implementation, Regulation, Procedural requirement 
SUMARIO: I. Primer contacto social con la figura del mediador. II. Implantación de la mediación. III. Bibliografía.

SUMMARY: I. First social contact with the figure of the mediator. II. Implementation of mediation. III. Bibliography.

"Se requieren nuevas formas de pensar para resolver los problemas creados por las viejas formas de pensar"

Albert Einstein

\section{PRIMER CONTACTO SOCIAL CON LA FIGURA DEL MEDIADOR}

De acuerdo con RODRÍGUEZ GARCÍA, la mediación es un método de gestión de conflictos que se caracteriza por la presencia de un tercero, el mediador, que ayuda a las partes a que encuentren la mejor solución o, al menos, la mejora de la situación que traen, objeto de disputa, debiendo ser esta solución mutuamente aceptable, teniendo en cuenta, además, la continuidad de las relaciones en el futuro. ${ }^{2}$ De ello podemos extraer, principalmente, que el mediador es un sujeto que debe limitarse a "ayudar a las partes a que encuentren la mejor solución", es decir, no debe imponer una solución a las partes, ni encontrarla por ellas, sino reconducir el diálogo para que quienes tengan un conflicto reconstruyan la relación que ostenten y den respuesta por sí mismos al problema. Por supuesto, palabras como "problema" o "conflicto" no deben interpretarse como un simple suceso de enfrentamiento, pues normalmente la mediación entrará en juego o tendrá cabida frente a disputas más complejas o prolongadas en el tiempo, que habrán de dar lugar a continuos desencuentros entre los sujetos implicados. Según PRUITT Y RUBIN, el conflicto puede definirse como la divergencia percibida de intereses $\mathrm{y}$ creencias que hace que las aspiraciones corrientes de las partes no puedan ser alcanzadas simultáneamente. ${ }^{3}$

Sin embargo, en los habituales foros de debate y entornos en los que se tratan estos conceptos, cabría escapar de la necesidad, tan siquiera casual, de exponer estas definiciones, pues es habitual para el jurista y el operador en el Derecho pensar en la resolución extrajudicial de conflictos como alternativa al litigio. Son muchas las revistas que mes a mes, o trimestralmente, recibimos de nuestros diversos colegios profesionales, bien como juristas o bien como colaboradores, y en muchas de ellas son habituales los artículos sobre novedades legislativas, jurisprudenciales o doctrinales, que de vez en cuando se dejan vislumbrar también en el campo de la mediación, por lo que nos es cercano, y hasta teóricamente cotidiano, el concepto de mediación. En el 
mismo sentido, en momentos de cambio pueden hacerse eco de manera efímera figuras como esta en telediarios y periódicos.

Sin embargo, el público objetivo de revistas y artículos científicos se suele circunscribir a quienes, como ya indicaba, por su oficio, ya conocen la figura de la mediación, y hasta puede ser que crean que dicha figura suponga una alternativa a la profesión del destinatario cuya práctica podría ser poco rentable para el mismo. La otra vertiente informativa mencionada, los medios de comunicación, carecen de permanencia o insistencia tratándose de cuestiones poco polémicas o llamativas para el público general, y se centran como sistema habitual en bombardear al lector/espectador con asuntos de rigurosa tendencia, por lo que rara vez van a suponer un medio divulgativo continuo que exponga ampliamente al gran público información sobre algún tema en concreto.

Por lo tanto, fuera del amplio espectro de sujetos que se mueven en el ámbito de trabajo de la resolución de conflictos, judicial o extrajudicial, nos encontramos con un gran desconocimiento general sobre la figura del mediador. No es difícil comprobar este hecho. Tan solo hay que pasear por cualquier calle o avenida y comparar las placas profesionales y rótulos de oficinas para comprobar la prácticamente nula presencia de alguna que diga exclusivamente "Mediador Civil". De hecho, como ha realizado quien suscribe, basta con salir a la calle y preguntar a la gente.

- ¿Sabe usted qué es un abogado?

- Sí, una persona que defiende los intereses de alguien en un juzgado.

- Y, sin embargo, ¿conoce usted la figura del mediador?

- Algo me suena, pero no sé lo que es.

- Y, si tuviera usted un conflicto, un daño por el que reclamar, por ejemplo, ¿acudiría a un abogado o a un mediador?

- Pues, evidentemente, a un abogado.

Esta es la reproducción de un encuestado o encuestada cualquiera, pero podría ser el diálogo mantenido con el ochenta por ciento de tales. Así es. Es lógico que, si no se conoce la figura del mediador, no se acuda a uno. Sin embargo, y aun cuando esta figura pueda ser conocida por el veinte por ciento restante (siempre en términos aproximados y absolutamente relativos a la estadística), sorprende conocer que, de esa porción menor de la ciudadanía hay quien, a pesar de saber que existe algo llamado mediación, acudirían a un abogado para resolver su conflicto. 
- ¿Por qué acudiría a un abogado?

- Bueno, no conozco a ningún mediador, sé que existe porque ayer vi uno en una película; pero sí que conozco a mi abogado habitual, y le tengo más confianza.

Por supuesto, hay quien conoce la figura del mediador, sabe lo que supuestamente hace. Pero esa parte del público piensa en el mediador como quien piensa en un gorila africano: sabe lo que es, visualiza vagamente la forma que tiene, ha visto en películas el ruido que hacen y cómo se comportan, pero nunca han visto uno en persona, ni tiene especial interés en conocerlo. Y ello, redundando en lo anterior, proviene del desconocimiento. Todo el mundo conoce al abogado, llevamos siglos trabajando con la gente, publicitándonos, proliferando. Salimos en programas, tertulias, películas, series, hasta en dibujos animados. Hay documentales sobre grandes y escandalosos pleitos de presidentes, celebridades, artistas, etc. ¿Quién no ha visto a Charlize Theron, Keanu Reeves y al gran Al Pacino en "El Abogado del Diablo"?

$\mathrm{Y}$, sin embargo, no hay grandes producciones sobre mediadores que se vean persuadidos por el ansia de poder ni distraídos alegremente por los súcubos. Ni series, ni tertulias. El ciudadano de a pie no sabe lo que es la mediación $\mathrm{y}$, como consecuencia, nadie busca que un mediador resuelva sus problemas. Es obvio, no hay grandes casos de mediación de los que se hagan eco los telediarios o periódicos; no existen, en esas mesas de análisis político que acercan la actualidad normativa al público general, mediadores especializados, como sí que hay abogados, periodistas, analistas y políticos. No se anuncian grandes despachos (o más bien instituciones) de mediación que ofrezcan al ciudadano soluciones económicas a sus problemas dinerarios, conyugales o laborales. Falta publicidad.

Llega tarde, además, el artículo 414 de la Ley de Enjuiciamiento Civil cuando, en la audiencia previa, en boca de su Señoría, se plantea (si acaso se menciona) la posibilidad de someterse a mediación, y poco habituales son los autos que dan un "frenazo procesal" al litigio en este punto para "ofrecer" la posibilidad de asistir a la sesión informativa. El proceso se encuentra iniciado, las partes ya han mostrado su jugada inicial en sus respectivos escritos procesales, los letrados ya han realizado la parte más sustancial de su trabajo, que es plasmar en papel el conflicto y los motivos que sostienen la postura de su cliente frente al mismo. Los clientes ya han removido cielo y tierra para encontrar ese escurridizo "papelito" que seguro va a suponer que la balanza se incline a su favor cuando su Señoría lo analice. E incluso, como es habitual, los profesionales que intervengan habrán ejercido con cautela la obligación 
de presentar a su defendido su hoja de encargo, a fin de recibir la pertinente provisión de fondos. En este punto, nos encontramos en el "anticlímax" del acuerdo, las partes no podrían estar más lejos de llegar a un entendimiento, ni mucho menos someterse a un proceso de mediación.

\section{IMPLANTACIÓN DE LA MEDIACIÓN}

Como en todo, hay excepciones. Como exponen UTRERA GUTIÉRREZ y PEÑA YÁÑEZ, ${ }^{5}$ en el Juzgado de Familia ( $\left({ }^{\mathrm{a}}\right.$ Instancia 5) de Málaga se viene practicando la mediación familiar incluso antes de publicarse la primera ley española en la materia, y desde el año 2000 vienen aplicando este sistema con la finalidad de fomentar la mediación familiar como método de resolución de los conflictos de familia. En los momentos iniciales, y dado que se carecía de Ley nacional de mediación y de legislación autonómica en esta materia (en Andalucía la ley de Mediación familiar no se promulga hasta el año 2009), el marco legal para poner en marcha su funcionamiento, y concretamente para invitar a las partes a que asistiesen a la primera sesión informativa presencial, se amparó en diversos preceptos de la Ley de Enjuiciamiento Civil (artículos 770, pfo. $5^{\circ}$ y $7^{\circ}$, y 771.2.) y del Código Civil (artículos 91, 92.5, 96, 97.1 y 103) que exteriorizan una clara opción del legislador por las soluciones autocompositivas en los conflictos de familia.

Ya desde 2006, incluso, podemos ver como bajo la dirección del Magistrado D. Pascual Ortuño se establece un protocolo para la mediación intrajudicial en juzgados de familia. Diversas magistradas y jueces, en aplicación de este protocolo en Juzgados de Familia de Barcelona ( $\left.n^{\circ} 18\right)$, Madrid ( $\left.n^{\circ} 29\right)$ Málaga $\left(n^{\circ} 5\right)$, Palma de Mallorca ( $\left.n^{\circ} 12\right)$ Pamplona ( $\left.n^{\circ} 3\right)$ y Sevilla $\left(n^{\circ} 7\right)$, han dado lugar a la resolución de los conflictos familiares, teniendo como principal impulsor del servicio de mediación a la figura del Juez, en colaboración con el resto de los agentes procesales participantes. ${ }^{6}$

A modo de ejemplo, fuera del ámbito familiar, y volviendo a ese "frenazo procesal" que mencionaba anteriormente, tenemos un Auto del Juzgado de Primera Instancia número 18 de Granada, que, en el marco de un proceso verbal civil de reclamación de cantidad por la suma de 59,19 €,en fecha 11 de noviembre de 2015, en su parte dispositiva, acuerda: "Con carácter previo a su admisión a trámite se convoca a las partes a una sesión informativa de mediación... debiendo comunicar a este juzgado la decisión adoptada al respecto". Resoluciones como esta ponen en el punto de mira el derecho a la tutela judicial efectiva, pues pueden ser interpretados como límites a dicho derecho si entendemos que se trata de una inadmisión a trámite de la demanda. 
Sin embargo, de acuerdo con CONFORTI, ${ }^{7}$ la inadmisión (que se traduce en la ineficacia total del acto defectuoso) no tiene el mismo significado jurídico que el no dar curso a la tramitación (que conlleva que el acto se admita y surta una serie de efectos aunque se suspenda su tramitación hasta que se subsane un defecto), y es que tanto la admisión como la inadmisión deben ser declaradas expresamente, cosa que no hace el auto referenciado. Pero el propio CONFORTI se pregunta ¿no siendo obligatoria por ley, la mediación previa a incoar acción judicial puede ser considerada un requisito procesal? Termina por interpretar que, en orden al interés público y por medio del ius cogens, cabe interpretar el intento de mediación como requisito de procedibilidad, no vulnerando en absoluto el derecho a la tutela judicial efectiva, siendo el método más deseable para resolver en conflicto, con arreglo a dar prioridad judicial a conflictos realmente graves para los que la sociedad debe dar una solución lo más inmediata posible, sin distraerse en problemas de baja litigiosidad.

Sin embargo, ello no se ha visto reflejado expresamente en la ley procesal, ni se ha planteado la inclusión de la resolución extrajudicial de conflictos de carácter obligatorio, hasta ahora. Y es que el Anteproyecto de Ley de Medidas de Eficiencia Procesal del Servicio Público de Justicia, ${ }^{8}$ publicado el 21 de enero de 2021, con ocasión de la celebración del Día Europeo de la Mediación, en el portal web del Ministerio de Justicia, dando inicio así el trámite de audiencia e información pública, implanta una serie de medidas procesales que darán lugar en su momento a la firme y verdadera obligatoriedad legal de pasar por la resolución extrajudicial de conflictos, entre cuyas formas se encuentra obviamente la mediación, como requisito de procedibilidad en el proceso judicial. Más no ha de entenderse esta obligatoriedad en sentido absoluto, y por ello transcribo a continuación el acotamiento en el orden civil que plantea el propio Anteproyecto en su artículo primero, realizando algunos apuntes:

"En el orden jurisdiccional civil, con carácter general, se considerará requisito de procedibilidad acudir previamente a algún medio adecuado de solución de controversias para que sea admisible la demanda".

No se encorseta la resolución extrajudicial de conflictos a la mediación, ni tan siquiera a las formas de resolución extrajudicial de conflictos más relevantes, sino que da cabida a una amplia posibilidad de negociación.

"Para entender cumplido este requisito habrá de existir una identidad entre el objeto de la negociación y el objeto del litigio, aun cuando las pretensiones sobre dicho objeto pudieran variar". 
Evidentemente, establece la necesidad de que el objeto del supuesto litigio se corresponda con el objeto de ese intento de resolución extrajudicial, se trate de la que se trate.

"Se considerará cumplido este requisito si se acude previamente a la mediación, a la conciliación o a la opinión neutral de un experto independiente, si se formula una oferta vinculante confidencial o si se emplea cualquier otro tipo de actividad negocial no tipificada legalmente pero que cumpla lo previsto en el apartado anterior y permita dejar constancia de la recepción por la parte requerida de la propuesta de negociación, así como de su fecha, contenido e identidad de la parte proponente. Singularmente, se considerará cumplido el requisito cuando la actividad negocial se desarrolle directamente por las partes asistidas de sus abogados cuando su intervención sea preceptiva".

Como mencionaba, se abre un abanico de posibilidades sin límite a la negociación, mientras se constate, al menos, que una parte ha tratado de comunicar fehacientemente a la otra su ofrecimiento de negociación. Este punto, por otro lado, podría llegar a ser modificado en el trámite de información pública, pues el concepto indeterminado "cualquier otro tipo de actividad negocial" podría dar lugar a la admisión de un simple burofax previo a la presentación de la demanda en que, además de advertir del inicio de actuaciones (como es habitual en orden a la buena fe procesal), se incluya una propuesta de acuerdo para salvar el requisito de procedibilidad, dejando sin efecto absolutamente toda la propuesta de reforma si ello termina derivando en la realización del litigio sin que se derive realmente a las partes a una alternativa de resolución extrajudicial (quizás ya en este punto intrajudicial) del conflicto.

"No obstante, no podrán ser sometidos a medios adecuados de solución de controversias, ni aun por derivación judicial, los conflictos que afecten a derechos y obligaciones que no estén a disposición de las partes en virtud de la legislación aplicable, sin perjuicio de que sí será posible su aplicación en relación con los efectos y medidas previstos en los artículos 102 y 103 del Código Civil. En ningún caso podrán aplicarse dichos medios de solución de controversias a los conflictos que versen sobre alguna de las materias excluidas de la mediación conforme a lo dispuesto en el artículo 87 ter de la Ley Orgánica 6/1985, de 1 de julio, del Poder Judicial. No se exigirá actividad negocial previa como requisito de procedibilidad cuando se pretenda iniciar un procedimiento para la tutela judicial civil de derechos fundamentales, la adopción de 
las medidas previstas en el artículo 158 del Código Civil y cuando se solicite autorización para el internamiento forzoso por razón de trastorno psíquico conforme a lo dispuesto en el artículo 763 de la Ley 1/2000, de 7 de enero, de Enjuiciamiento Civil".

En este punto, salva del requisito de procedibilidad algunas materias limitadas para la libre disposición, y por supuesto mantiene los campos jurídicos vetados ya anteriormente para la mediación.

En todo caso, dicho todo esto, y volviendo sobre nuestros pasos, hay que tener en cuenta que la implantación como requisito no supondrá por sí misma el éxito de la resolución extrajudicial de conflictos. Y es que, como indicaba al inicio, si bien la incardinación de la mediación (u otra alternativa) de manera previa al proceso, hará que la negociación llegue a su debido tiempo al conflicto, antes de realizar incluso otros esfuerzos profesionales que podrían resultar vanos, resulta también relevante educar a la ciudadanía en el uso y las virtudes de la mediación, e incluso dar publicidad a su figura de formas tan cotidianas como se les da participación social a otros profesionales (tal como a los abogados, como ejemplificaba), pues de lo contrario toda la implementación del anteproyecto acabará reducida a un párrafo más en el burofax previo a la demanda. Ello es así porque, si a las partes no se les exponen las ventajas de la mediación, y las desconocen, acudirán a este primer acto es una posición cerrada, defensiva y hermética, impermeable a toda posibilidad de acuerdo que pudiera darse, y finalizará el intento en el acto. Y ello se puede deducir si introducimos a toda la reforma planteada a uno de los conceptos y presupuestos básicos de la mediación: la voluntariedad, ${ }^{9}$ que, tal como indica PALANDRI, es la primera clave que debe darse para que se abra la vía de la mediación, y ello no solo porque no puede imponerse participar en un procedimiento donde la comunicación debe ser fluida y libre, sino porque, lógicamente, no podemos llamar "acuerdo" a una disposición alcanzada por imposición.

Debemos partir de que el hecho de acudir a la mediación es voluntario, así como permanecer en el proceso mismo. Acceder al proceso de mediación de manera forzada, como requisito para poder continuar (o iniciar) el litigio, pasa por encima de dicha voluntariedad ya que, aunque no se obligue al implicado a permanecer en la mediación, sí que se le obliga a pasar por el intento. Ello no supone, sin embargo, una violación de las normas de la mediación como tal, pues no tiene por qué proseguir más allá de la sesión informativa, y de hecho tendrían igual regulación tanto la obligación de acudir a sesión informativa como su carácter voluntario. Pero sí que desvirtúa el inicio del proceso mediador en el sentido de provocar en el obligado una aversión al 
propio concepto de mediación, por mera imposición del mismo, lo que dificulta aun más el poder alcanzar un acuerdo. Sin embargo, tal vez este sea el precio a pagar para la consecución de la implantación real de la resolución extrajudicial de conflictos en nuestra sociedad.

\section{CONCLUSIÓN}

Queda mucho por andar en el campo de la resolución extrajudicial de conflictos. Aun no se ha elevado a rango Ley lo que ahora es un anteproyecto, pero sí que hemos visto a la mediación alcanzar rango de Ley a nivel estatal y autonómico, y pasada prácticamente una década aun no es notoria su aplicación. Tal vez no suframos falta de regulación, pues se encuentra regulada hace tiempo.

Puede que, más bien, suframos falta de publicidad. La sociedad española precisa de un desahogo inmediato de los órganos judiciales, es una imperiosa necesidad la que sufrimos en ese aspecto, y eso sí que es parte del acervo popular a todos los niveles. Todo ciudadano sabe que la Justicia es lenta, sin embargo, no todo ciudadano topa con esa lentitud ni se ve envuelto en procesos judiciales. No es un conocimiento adquirido por experiencia propia de cada individuo.

Tal vez sea el momento de acompañar las reformas legislativas con campañas de divulgación, talleres informativos, series televisivas. Tal vez sea el momento de mostrar a la sociedad para qué sirve el Mediador.

\section{BIBLIOGRAFÍA}

Anteproyecto de Ley de Medidas de Eficiencia Procesal del Servicio Público de Justicia, https://www.mjusticia.gob.es/es/AreaTematica/ActividadLegisl ativa/Documents/APL\%20Eficiencia\%20Procesal.pdf.

CONFORTI, O. D. F. (2016), “Análisis al Auto sobre la mediación intrajudicial como requisito de procedibilidad", Revista de Mediación, vol. 9, nº 2, http $\mathrm{s}: / /$ revistademediacion.com.

PALANDRI, E. (2018), Mediación: Manual de Formación Básica, Alveroni Ediciones, Córdoba, $2^{\mathrm{a}}$ edición.

PRUITT, D. G. y RUBIN, J. Z. (2004), Mediación para resolver con\#ictos, Ad-Hoc, Buenos Aires.

RODRÍGUEZ GARCÍA, C. (2016), Manual de Mediación Civil y Mercantil, Universidad Internacional de La Rioja, S. A. (UNIR), Logroño.

Revista de Estudios Jurídicos, UJAEN, España |AÑO 2021 NUM. 21, e6783 | ISSN 1576-124X 
UTRERA GUTIÉRREZ, J. L., MARTÍN NÁJERA, T. y PÉREZ SALAZAR, M. (2009), "Protocolo para la implantación de la mediación familiar intrajudicial en los juzgados y tribunales que conocen de procesos de familia", Revista de Mediación, $\mathrm{n}^{\circ}$ 4, https://revistademediacion.com.

UTRERA GUTIÉRREZ, J. L. y PEÑA YÁÑEZ, M. A. (2014), "El Servicio de Mediación Familiar Intrajudicial de los juzgados de familia de Málaga", Revista de Mediación, vol. 7, $\mathrm{n}^{\mathrm{o}}$ 1, https://revistademediacion.com.

\section{NOTAS}

1 Abogado del Ilustre Colegio de Abogados de Jaén. Dirección: franciscojesuscanovera@gmail.com.

2 RODRÍGUEZ GARCÍA, C. (2016), Manual de Mediación Civil y Mercantil, Universidad Internacional de La Rioja, S. A. (UNIR), Logroño.

3 PRUITT, D. G. y RUBIN, J. Z. (2004), Mediación para resolver conflictos, Ad-Hoc, Buenos Aires.

4 TAYLOR, H. (Director) \& KOPELSON, A. (Productor) (1997), The Devil's Advocate. Estados Unidos; Regency Enterprises [Película].

5 UTRERA GUTIÉRREZ, J. L. y PEÑA YÁÑEZ, M. A. (2014), "El Servicio de Mediación Familiar Intrajudicial de los juzgados de familia de Málaga”, Revista de Mediación, vol. 7, ${ }^{\circ}$ 1, https://revistademediacion.com.

6 UTRERA GUTIÉRREZ, J. L., MARTÍN NÁJERA, T. y PÉREZ SALAZAR, M. (2009), "Protocolo para la implantación de la mediación familiar intrajudicial en los juzgados y tribunales que conocen de procesos de familia", Revista de Mediación, $\mathrm{n}^{\circ} 4$, https://revistade mediacion.com.

7 CONFORTI, O. D. F. (2016), “Análisis al Auto sobre la mediación intrajudicial como requisito de procedibilidad”, Revista de Mediación, vol. 9, nº 2, https://revistademediacion.com.

8 Anteproyecto de Ley de Medidas de Eficiencia Procesal del Servicio Público de Justicia, https://www.mjusticia.gob.es/es/AreaTematica/ActividadLegislativa/Documents/AP L\%20Eficiencia\%20Procesal.pdf.

9 PALANDRI, E. (2018), Mediación: Manual de Formación Básica, Alveroni Ediciones, Córdoba, $2^{\mathrm{a}}$ edición. 